Gender comparison of online and land-based gamblers from a nationally representative sample : Does gambling online pose elevated risk?

\title{
Edgren, Robert
}

2017-07

Edgren , R , Castren , S , Alho , H \& Salonen , A H 2017 , ' Gender comparison of online and land-based gamblers from a nationally representative sample : Does gambling online pose elevated risk? ' , Computers in Human Behavior, vol. 72 , pp. 46-56 . https://doi.org/10.1016/j.chb.2017.02.033

http://hdl.handle.net/10138/235609

https://doi.org/10.1016/j.chb.2017.02.033

unspecified

publishedVersion

Downloaded from Helda, University of Helsinki institutional repository.

This is an electronic reprint of the original article.

This reprint may differ from the original in pagination and typographic detail.

Please cite the original version. 
Full length article

\title{
Gender comparison of online and land-based gamblers from a nationally representative sample: Does gambling online pose elevated risk?
}

\author{
Robert Edgren ${ }^{\mathrm{a}, \mathrm{b},{ }^{*} \text {, Sari Castrén }}{ }^{\mathrm{c}, \mathrm{d}}$, Hannu Alho ${ }^{\mathrm{e}, \mathrm{c}}$, Anne H. Salonen ${ }^{\mathrm{c}, \mathrm{d}}$ \\ ${ }^{a}$ Institute of Behavioral Sciences, University of Helsinki, Siltavuorenpenger 1 A (P.O. Box 9), Finland \\ ${ }^{\mathrm{b}}$ National Institute for Health and Welfare, Department Tobacco, Gambling and Addiction, P.O. Box 30, FI-00271, Helsinki, Finland \\ ${ }^{\mathrm{c}}$ National Institute for Health and Welfare, Department of Public Health Solutions, The Alcohol, Drugs and Addictions Unit, P.O. Box 30, FI-00271, Helsinki, \\ Finland \\ ${ }^{\mathrm{d}}$ Institute of Clinical Medicine, University and University Hospital of Helsinki, Finland \\ e Abdominal Center, University and University Hospital of Helsinki, Finland
}

\section{A R T I C L E I N F O}

\section{Article history:}

Received 21 November 2016

Received in revised form

2 February 2017

Accepted 9 February 2017

Available online 10 February 2017

\section{Keywords}

Online gambling

Gaming

Gender comparison

Risk factors

National survey

\begin{abstract}
A B S T R A C T
The expansion of online gambling opportunities calls for better comprehension of online gambling, including relevant gender specific correlates. This study compared online and land-based gamblers among males and females separately, utilizing a nationally representative Finnish survey sample of 18 -74 year olds. Online gamblers were younger than land-based gamblers and had full-time working status more often than land-based gamblers, with partial indication of land-based gamblers' monthly income being lower. Online gambling was associated with participation in computer or video gaming more strongly than with land-based gambling. Results show that the strongest predictors of online gambling common to both genders were younger age, computer gaming and gambling on multiple gambling types. Risky alcohol consumption and tobacco smoking were not associated to gambling mode when controlling for other factors. Results indicate that particularly for females online gambling may be related to higher relative expenditure and at-risk and problem gambling, providing implications for tailored interventions. The continued study of subgroups of gamblers is necessary to comprehensively understand the altering gambling milieu.
\end{abstract}

๑ 2017 Elsevier Ltd. All rights reserved.

\section{Introduction}

Online gambling is unique due to around the clock internet access almost everywhere. Also as a growing industry, online gambling opportunities are continuously increasing. Being cornerstone ecological factors, increased availability and accessibility of online gambling may have considerable influence on the prevalence of at-risk and problem gambling, as suggested by the Pathways model (Blaszczynski \& Nower, 2002). Studying gambling

Abbreviations: LB, land-based; AUDIT-C, Alcohol Use Disorder Identification Test -Consumption; PGSI, Problem Gambling Severity Index; MHI-5, 5 item Mental Health Inventory; OR, odds ratio; CI, confidence interval; SD, standard deviation; OSF, Official Statistics of Finland.

* Corresponding author. Institute of Behavioral Sciences, University of Helsinki, Siltavuorenpenger 1 A (P.O. Box 9), Finland.

E-mail addresses: robert.edgren@helsinki.fi (R. Edgren), sari.castren@thl.fi (S. Castrén), hannu.alho@thl.fi (H. Alho), anne.salonen@thl.fi (A.H. Salonen). mode (i.e. online and land-based), known risky behaviors and gambling-related factors are thus of paramount importance. Here online gambling refers to gambling that takes place over the internet, while land-based gambling refers to brick-and-mortar based services.

\subsection{Demographics associated to gambling mode}

Online gambling tends to be more common among males, and land-based gambling more common among females (Gainsbury, Russell, Blaszczynski, \& Hing, 2015; Gainsbury, Wood, Russell, Hing, \& Blaszczynski, 2012; Kairouz, Paradis, \& Nadeau, 2012; McBride \& Derevensky, 2009). Nonetheless, online gambling may be more appealing to females than land-based gambling because of its anonymity and less stigmatizing nature (Corney \& Davis, 2010; McCormack, Shorter, \& Griffiths, 2014). Strictly land-based gamblers have been found to be older than those who gamble online 
(Gainsbury, Russell, Blaszczynski, et al., 2015; Kairouz et al., 2012. McBride \& Derevensky, 2009), although age related differences have also been concluded to not prevail (Gainsbury et al., 2012). Online gambling has been associated to being married (Gainsbury et al., 2012; Svensson \& Romild, 2011) but also to a lower likelihood of being married (Wood \& Williams, 2011). Gambling on both online and land-based platforms has been associated to a higher likelihood of working full-time (Gainsbury, Russell, Blaszczynski, et al., 2015; Gainsbury et al., 2012) and online gambling to higher income level (Gainsbury et al., 2012; Wood \& Williams, 2011) compared to strictly land-based gamblers. On the contrary being a student or unemployed, and particularly for females having low level education and low income have also been associated to online gambling (Gainsbury et al., 2012; Svensson \& Romild, 2011).

\subsection{Risky behaviors, perceived mental health and gambling mode}

The association between online gambling and substance use is somewhat ambiguous. Tobacco, alcohol and illicit drug use were concluded more common among online gamblers than land-based gamblers (Kairouz et al., 2012; Wood \& Williams, 2011). In contrast, land-based gamblers have been found to have more gambling related health and psychological problems than online gamblers (Gainsbury, Russell, Hing, Wood, \& Blaszczynski, 2013). While controlling for demographic and gambling-related factors, alcohol and drug use have been found to not be associated to either mode of gambling (Gainsbury et al., 2012).

Gaming is a progressively popular pastime, which for some may become harmful (Ferguson, Coulson, \& Barnett, 2011). Here gaming refers to any form of computer or video gaming. Overlap in gaming and gambling is becoming evident, and online gambling should not be viewed in isolation, as there is an increasing amount of multimedia integration (Griffiths \& Parke, 2010). Freemium games that mimic gambling without monetary stakes, social casino games, have been studied in relation to monetary gambling with results suggesting that gamers may migrate to gambling (Elton-Marshall, Leatherdale, \& Turner, 2016; Gainsbury, Russell, \& Hing, 2014; Gainsbury, Russell, King, Delfabbro, \& Hing, 2016).

Research has yet to elucidate how gaming in general and gambling are associated with each other. There are similarities between problematic forms of gaming and gambling suggesting that Internet Gaming Disorder could be considered a behavioral addiction along with Gambling Disorder (APA, 2013; Duven, Müller, Beutel, \& Wölfling, 2015; Petry \& O'Brien, 2013) but very little research has been conducted on this subject (Baggio, Gainsbury, Berchtold, \& Iglesias, 2016; Király et al., 2014). Nonetheless, research suggests frequent gaming to be associated to disordered gambling among Finnish adolescents (Castrén, Grainger, Lahti, Alho, \& Salonen, 2015). Also, a positive association has been found between the frequency of online gambling and online gaming (Floros, Siomos, Fisoun, \& Geroukalis, 2013). Importantly, adolescent online gamblers have been found to display higher levels of problematic internet use and problematic gambling compared to land-based gamblers, even when controlling for the number of gambling games engaged in (Baggio et al., 2016). Few studies have investigated gaming and gambling among older adults (Ariyabuddhiphongs, 2012). Being based on similar interfaces, it is intuitive that online gambling and gaming may co-occur. What is less clear is whether gaming which is excessive or otherwise perceived as detrimental is even more strongly associated to online gambling; some findings suggest an association with mental health problems (Hopley \& Nicki, 2010; Petry \& Weinstock, 2007; ScholesBalog \& Hemphill, 2012; Wood, Griffiths, \& Parke, 2007) while others argue against it (Wood \& Williams, 2011).

\subsection{Gambling-related factors associated to gambling mode}

Previous studies show that gambling-related factors (i.e. frequency, number of gambling types, gambling expenditure, and severity of gambling) often have the strongest associations with online gambling. Compared to land-based gamblers, online gamblers tend to gamble more frequently, gamble on a larger amount of gambling types and spend more money on gambling (EltonMarshall et al., 2016; Gainsbury, Russell, Hing, et al., 2015; Gainsbury, Russell, Blaszczynski, et al., 2015; Gainsbury et al., 2012, 2013; Kairouz et al., 2012; Wardle, Moody, Griffiths, Orford, \& Volberg, 2011). Additionally, males have been found to gamble on several online gambling types regularly more often than females (McCormack et al., 2014). Financial harms are a core aspect of gambling related harm (Langham et al., 2015), where viewing expenditure proportionately to income may provide added value (Currie et al., 2006). Spending a higher proportion of income on gambling is associated to gambling problems (Currie et al., 2006; Weinstock, Whelan, \& Meyers, 2008). Examining expenditure by income has not been compared for online and land-based gamblers to the knowledge of the authors. Considering continuous forms of play and ease of payment, it is plausible that online gamblers end up spending more than their strictly land-based gambling counterparts.

Online gamblers (who often also gamble in land-based venues) have been shown to display at-risk and problem gambling more frequently than their land-based gambling counterparts (EltonMarshall et al., 2016; Gainsbury, Russell, Blaszczynski, et al., 2015; Gainsbury et al., 2013; Kairouz et al., 2012; McBride \& Derevensky, 2009; Wood \& Williams, 2011). It remains somewhat unclear what the relationship is between at-risk and problem gambling and the internet as a mode of gambling. Is the internet intrinsically a detrimental mode for gambling, or do gamblers with elevated risk for developing gambling problems tend to use the internet for gambling more often than non-problem gamblers? Evidence is available that suggests it is not the internet in itself that is harmful, but rather the result of elevated overall engagement in gambling (Gainsbury et al., 2013; Wardle et al., 2011). On the other hand features of online gambling give cause for concern, for example continuous access and anonymity. Thus as a mode of gambling, the internet may assist the progression of gambling problems amongst those at elevated risk (Gainsbury et al., 2013) which would be in line with the conceptualization of the Pathways Model (Blaszczynski \& Nower, 2002).

\subsection{The Finnish gambling context}

Gambling opportunities and advertisements are vastly evident in Finland. Gambling in Finland is government regulated with a monopoly. Up until December 2016, the monopoly has been distributed across three providers, all of which have had the right to arrange gambling services on the internet (Lotteries Act, $14 \S$ 20.5.2011/575). The proportion of gambling that takes place on the internet is growing nationally (Salonen \& Raisamo, 2015). For example, in 2015 Veikkaus Oy's (provides e.g. sports betting and lottery services in Finland) revenue was 2095.3 million euros, out of which $43.3 \%$ was generated from online gambling (Veikkaus, 2016). Based on these internal reports, the share of revenue generated by online gambling increased by 6\% since 2013 (Veikkaus, 2016, 2014).

\subsection{The current study}

Online and land-based gamblers have not previously been compared in the Finnish context, and little is known about the gender specific characteristics. Gender specific links of problematic 
gambling occurring online has been noted as a research area needing more attention (McCormack et al., 2014). Furthermore there is a dearth of evidence evaluating the convergence of gambling and computer gaming (Gainsbury et al., 2016), and previous research on the modes of gambling have often utilized school-based and online samples (Gainsbury, Russell, Blaszczynski, et al., 2015; Gainsbury et al., 2012; King, Delfabbro, Kaptsis, \& Zwaans, 2014; McCormack et al., 2014). Duly, utilizing a nationally representative random sample, this study investigates the gender specific distinctions between online gamblers and landbased gamblers in three domains: demographic characteristics (1), risky behaviors (referring here to tobacco smoking, alcohol consumption and gaming) and perceived mental health (2), and gambling-related factors (number of gambling types gambled; expenditure by net income; severity of gambling problems) (3).

\section{Method}

\subsection{Sample and procedure}

Data was extracted from the cross-sectional Finnish Gambling 2015 survey (Salonen \& Raisamo, 2015). The survey was conducted in accordance with the ethical standards of the Declaration of Helsinki. See Salonen, Alho and Castrén's (2016) publication for sampling methods. All participants that may gamble legally $(\geq 18$ years old) and had gambled within the past 12 months were selected for the present study ( $\mathrm{n}=3555,46.2 \%$ female). $34.2 \%$ of male and $22.1 \%$ of female gamblers had gambled online in the past 12 months. $47.5 \%$ of male and $42.2 \%$ of female gamblers had taken part in computer/video gaming in the past 12 months.

\subsection{Measures}

\subsubsection{Gambling-related factors}

Participants were asked whether they had gambled on 18 predefined gambling types (yes/no). These items were used to calculate how many gambling types each participant engages in. Participants who reported engaging in some form of online gambling within the past 12 months were classified as online gamblers, while the remaining gamblers were classified as strictly land-based gamblers. Online gambling has previously been operationalized in the same manner (Baggio et al., 2016; Gainsbury, Russell, \& Hing, 2014; Gainsbury et al., 2012). Gambling types were not utilized in this study because online gambling types were not inquired as comprehensively as land-based gambling types. The survey nonetheless included a dichotomous question concerning online gambling in general, assuring that no online gamblers went unidentified.

Participants were asked how much money they spend on gambling on average per week. Expenditure on gambling and reported net income was used to calculate the percentage of income each participant used on gambling. Weekly expenditure was converted to monthly expenditure, accounting for the frequency of gambling. Expenditure by net income was grouped into $<1 \%$ and $\geq 1 \%$. This classification has been used previously (Currie et al., 2006). Reporting income equal to zero $(n=54)$ was recoded as a missing value. One outlier for weekly gambling expenditure was identified $(50,000 €)$ and was replaced with the second-to-highest value $(1500 €)$.

At-risk and problem gambling was evaluated with the 9 item Problem Gambling Severity Index (PGSI; Ferris \& Wynne, 2001). The PGSI measures gambling behavior and related consequences with response options never ( 0 ), sometimes, most of the time, and almost always (3). The sum score ranges from 0 to 27 , where a score of 0 indicates no problem, 1-2 low risk gambling, 3-7 moderate risk gambling and 8-27 problem gambling. In this sample the average PGSI score was $0.45(\mathrm{SD}=1.61)$ for males and 0.20 $(S D=0.93)$ for females. The PGSI has been concluded to be unidimensional, internally consistent and have adequate itemresponse characteristics (Orford, Wardle, Griffiths, Sproston, \& Erens, 2010). The PGSI displayed good internal consistency in the current sample (Cronbach's alpha $=0.83$ ).

\subsubsection{Tobacco and alcohol use}

Past year tobacco smoking was inquired with a single question with 3 response options: daily, occasionally, and not at all. Alcohol consumption was determined with the three item Alcohol Use Disorders Identification Test -Consumption (AUDIT-C; Bush, Kivlahan, McDonell, Fihn, \& Bradley, 1998). Cronbach's alpha was 0.62 . The sum score of the AUDIT-C ranges from 0 to 12 (items scored $0-4$ ), in which a higher score indicates riskier consumption. Risky consumption was defined as scoring 5 or more for females and 6 or more for males (Edgren, Castrén, Jokela, \& Salonen, 2016; Kaarne, Aalto, Kuokkanen, \& Seppä, 2010). AUDIT-C is capable of detecting risky consumption of alcohol in the general population, and using gender specific cut-offs improves classification accuracy (Dawson, Grant, Stinson, \& Zhou, 2005).

\subsubsection{Gaming}

Gaming was evaluated with the following question: "Have you played video, console, computer or mobile games in the past 12 months?" with two answer options (yes/no). Positive responses lead to the follow-up question "In the past 12 months how often have you felt that video, console, computer or mobile gaming may have caused you problems" with 4 response options: never, sometimes, often, and almost always. For analyses participants were classified as non-gamers, gamers who have not perceived problems (gaming without problems) and gamers who have perceived problems at least sometimes (gaming with probable problems).

\subsubsection{Perceived mental health}

Mental health was evaluated with the five-item Mental Health Inventory (MHI-5; Veit \& Ware, 1983). Items (scored 1-6) concern feelings of nervousness, calmness, happiness and feeling down and gloomy over the past 4 weeks. Sum scores are linearly transformed to vary from 0 to 100 , where high scores indicate good perceived mental health. A cut-off score of 52 was used to classify poor perceived mental health. The MHI-5 is valid for measuring mood and anxiety disorders, where a cutoff score of 52 functions adequately (Rumpf, Meyer, Hapke, \& John, 2001; Strand, Dalgard, Tambs, \& Rognerud, 2003). In this sample Cronbach's alpha for the MHI-5 was 0.79 .

\subsubsection{Demographics}

Information on gender and age were retrieved from the Statistics Finland register. Employment, marital status and net income were disclosed in the survey. Here, being single includes not being in a registered relationship. Monthly net income $(€)$ was grouped into quartiles for analysis: 0-1199, 1200-1799, 1800-2499 and $\geq 2500$.

\subsection{Statistical analyses}

Omnibus chi-square tests were conducted for males and females separately, analyzing whether differences were evident between strictly land-based gamblers and online gamblers on behalf of demographic characteristics (Table 1), risky behaviors and perceived mental health (Table 2), and gambling-related factors (Table 3). When the omnibus chi-square test indicated a statistically 
Table 1

Demographic characteristics by gambling mode reported in column percentages (\%) for weighted sample and sample size (n) for unweighted sample.

\begin{tabular}{|c|c|c|c|c|c|c|c|c|c|c|c|}
\hline & & \multicolumn{5}{|c|}{ Males $(n=1911)$} & \multicolumn{5}{|c|}{ Females $(n=1644)$} \\
\hline & & \multicolumn{2}{|c|}{$\begin{array}{l}\text { Online } \\
\text { gamblers } \\
(\mathrm{n}=653)\end{array}$} & \multicolumn{2}{|c|}{$\begin{array}{l}\mathrm{LB}^{\mathrm{b}} \\
\text { gamblers } \\
(\mathrm{n}=1258)\end{array}$} & \multirow[t]{2}{*}{$\mathrm{X}^{2}$ statistics } & \multicolumn{2}{|c|}{$\begin{array}{l}\text { Online } \\
\text { gamblers } \\
(\mathrm{n}=363)\end{array}$} & \multicolumn{2}{|c|}{$\begin{array}{l}\mathrm{LB}^{\mathrm{b}} \\
\text { gamblers } \\
(\mathrm{n}=1281)\end{array}$} & \multirow[t]{2}{*}{$\mathrm{X}^{2}$ statistics } \\
\hline & & $\mathrm{n}$ & $\%$ & $\mathrm{n}$ & $\%$ & & $\mathrm{n}$ & $\%$ & $\mathrm{n}$ & $\%$ & \\
\hline \multirow[t]{6}{*}{ Age group (years) } & $18-24$ & 73 & 13.2 & 127 & 12.4 & \multirow[t]{6}{*}{$\mathrm{X}^{2}(5, \mathrm{~N}=1875)=133.54, \mathrm{p}<0.001, \Phi \mathrm{c}=0.267$} & 27 & 10.4 & 98 & 10.8 & \multirow[t]{6}{*}{$\mathrm{X}^{2}(5, \mathrm{~N}=1671)=47.43, \mathrm{p}<0.001, \Phi \mathrm{c}=0.168$} \\
\hline & $25-34$ & 147 & $26.6^{\wedge}$ & 125 & 12.6 & & 86 & $24.4^{\wedge}$ & 171 & 14.4 & \\
\hline & $35-44$ & 150 & $21.8^{\wedge}$ & 181 & 14.8 & & 71 & $21.5^{\wedge}$ & 161 & 14.7 & \\
\hline & $45-54$ & 134 & 18.8 & 235 & 18.4 & & 61 & 16.4 & 219 & 17.6 & \\
\hline & $55-64$ & 104 & $14.1^{v}$ & 300 & 22.5 & & 74 & 17.9 & 328 & 23.5 & \\
\hline & $65-74$ & 45 & $5.5^{\vee}$ & 290 & 19.3 & & 44 & $9.4^{2}$ & 304 & 19.0 & \\
\hline \multirow[t]{9}{*}{ Employment status } & Work full-time & 407 & $63.6^{\wedge}$ & 491 & 40.9 & \multirow[t]{9}{*}{$\mathrm{X}^{2}(8, \mathrm{~N}=1872)=115.06, \mathrm{p}<0.001, \Phi \mathrm{c}=0.248$} & 170 & 48.3 & 547 & 45.2 & \multirow[t]{9}{*}{$\mathrm{X}^{2}(8, \mathrm{~N}=1670)=18.50, \mathrm{p}=0.018, \Phi \mathrm{c}=0.105$} \\
\hline & Work part-time & 17 & 2.7 & 36 & 3.1 & & 28 & 8.1 & 88 & 7.5 & \\
\hline & Entrepeneur & 63 & 9.2 & 146 & 11.5 & & 17 & 4.4 & 63 & 4.8 & \\
\hline & Student & 41 & 7.3 & 86 & 8.3 & & 29 & 9.1 & 76 & 7.6 & \\
\hline & Unemployed/Laid off & 41 & 6.2 & 87 & 7.3 & & 22 & 6.2 & 59 & 4.7 & \\
\hline & Retired (age) & 59 & $7.3^{\mathrm{v}}$ & 338 & 23.2 & & 58 & $13.0^{\circ}$ & 346 & 22.2 & \\
\hline & Retired (illness) & 17 & 2.4 & 53 & 3.9 & & 17 & 4.4 & 47 & 3.4 & \\
\hline & Homemaker & 0 & $0^{\mathrm{a}}$ & 1 & $0.1^{\mathrm{a}}$ & & 20 & 6.0 & 48 & 4.1 & \\
\hline & Missing/Other ${ }^{c}$ & 8 & 1.3 & 20 & 1.7 & & 2 & 0.5 & 7 & 0.5 & \\
\hline \multirow[t]{3}{*}{ Marital status } & Single $^{d}$ & 297 & $49.5^{\wedge}$ & 422 & 37.0 & \multirow[t]{3}{*}{$\mathrm{X}^{2}(2, \mathrm{~N}=1873)=27.75, \mathrm{p}<0.001, \Phi \mathrm{C}=0.122$} & 133 & 39.7 & 368 & 32.8 & \multirow[t]{3}{*}{$\mathrm{X}^{2}(2, \mathrm{~N}=1670)=6.61, \mathrm{p}=0.037, \Phi \mathrm{c}=0.063$} \\
\hline & Married & 295 & $42.2^{\mathrm{v}}$ & 689 & 52.2 & & 166 & 43.6 & 665 & 49.7 & \\
\hline & Separated/widowed & 61 & 8.3 & 147 & 10.8 & & 64 & 16.6 & 248 & 17.5 & \\
\hline \multirow[t]{5}{*}{ Net income $(€)$} & 1. Quartile (0-1199) & 78 & $12.6^{\vee}$ & 224 & 18.9 & \multirow[t]{5}{*}{$\mathrm{X}^{2}(4, \mathrm{~N}=1872)=36.74, \mathrm{p}<0.001, \Phi \mathrm{c}=0.140$} & 78 & 22.3 & 303 & 23.7 & \multirow[t]{5}{*}{$\mathrm{X}^{2}(4, \mathrm{~N}=1670)=3.37, \mathrm{p}=0.498, \Phi \mathrm{c}=0.045$} \\
\hline & 2. Quartile (1200-1799) & 84 & 13.1 & 240 & 18.1 & & 98 & 25.9 & 362 & 27.4 & \\
\hline & 3. Quartile (1800-2499) & 194 & $29.8^{\wedge}$ & 279 & 22.5 & & 112 & 31.3 & 349 & 28.0 & \\
\hline & 4: Quartile (2500-) & 269 & 40.5 & 427 & 33.7 & & 59 & 16.3 & 190 & 15.2 & \\
\hline & Missing & 28 & 4.0 & 88 & 6.8 & & 16 & 4.1 & 77 & 5.8 & \\
\hline
\end{tabular}

Observed cell count significantly higher $\left({ }^{\wedge}\right)$ or lower $\left({ }^{\vee}\right)$ than expected $(\mathrm{p}<0.01)$.

a Expected cell count less than 5; Фc estimate of effect size (Cramer's V).

b Land-based.

Includes military/community service, other, and does not want to disclose.

${ }^{\mathrm{d}}$ Includes participants not in a registered relationship. 


\begin{tabular}{|c|c|c|c|c|c|c|c|c|c|c|c|}
\hline & & \multicolumn{5}{|c|}{ Males $(\mathrm{n}=1911)$} & \multicolumn{5}{|c|}{ Females $(\mathrm{n}=1644)$} \\
\hline & & \multicolumn{2}{|c|}{$\begin{array}{l}\text { Online } \\
\text { gambling } \\
(\mathrm{n}=653)\end{array}$} & \multicolumn{2}{|c|}{$\begin{array}{l}\mathrm{LB}^{\mathrm{b}} \\
\text { gambling } \\
(\mathrm{n}=1258)\end{array}$} & \multirow[t]{2}{*}{$\mathrm{X}^{2}$ statistics } & \multicolumn{2}{|c|}{$\begin{array}{l}\text { Online } \\
\text { gambling } \\
(\mathrm{n}=363)\end{array}$} & \multicolumn{2}{|c|}{$\begin{array}{l}\mathrm{LB}^{\mathrm{b}} \\
\text { gambling } \\
(\mathrm{n}=1281)\end{array}$} & \multirow[t]{2}{*}{$\mathrm{X}^{2}$ statistics } \\
\hline & & $\mathrm{n}$ & $\%$ & $\mathrm{n}$ & $\%$ & & $\mathrm{~N}$ & $\%$ & $\mathrm{n}$ & $\%$ & \\
\hline \multirow[t]{4}{*}{ Tobacco smoking } & Daily & 159 & 24.8 & 266 & 21.2 & \multirow[t]{4}{*}{$\mathrm{X}^{2}(3, \mathrm{~N}=1873)=10.73, \mathrm{p}=0.013, \Phi \mathrm{c}=0.076$} & 72 & 20.1 & 181 & 14.2 & \multirow{4}{*}{$\mathrm{X}^{2}(3, \mathrm{~N}=1670)=10.66, \mathrm{p}=0.014, \Phi \mathrm{c}=0.080$} \\
\hline & Occasional & 130 & 21.6 & 205 & 17.8 & & 42 & 12.8 & 125 & 10.7 & \\
\hline & Never & 363 & 53.5 & 780 & 60.5 & & 249 & 67.2 & 974 & 75.0 & \\
\hline & Missing & 1 & $0.1^{\mathrm{a}}$ & 7 & $0.5^{\mathrm{a}}$ & & 0 & $0.0^{\mathrm{a}}$ & 1 & $0.2^{\mathrm{a}}$ & \\
\hline \multirow[t]{4}{*}{ Alcohol use ${ }^{c}$} & No consumption & 35 & 5.0 & 108 & 8.0 & \multirow{4}{*}{$\mathrm{X}^{2}(3, \mathrm{~N}=1875)=20.56, \mathrm{p}<0.001, \Phi \mathrm{c}=0.105$} & 34 & 9.1 & 162 & 11.7 & \multirow[t]{4}{*}{$\mathrm{X}^{2}(3, \mathrm{~N}=1671)=8.66, \mathrm{p}=0.034, \Phi \mathrm{c}=0.072$} \\
\hline & Non-risky consumption & 385 & 58.0 & 805 & 63.5 & & 266 & 73.0 & 975 & 75.9 & \\
\hline & Risky consumption & 227 & $36.1^{\wedge}$ & 326 & 27.0 & & 62 & 17.6 & 141 & 12.2 & \\
\hline & Missing & 6 & 0.9 & 19 & 1.5 & & 1 & $0.3^{\mathrm{a}}$ & 3 & $0.2^{\mathrm{a}}$ & \\
\hline \multirow[t]{4}{*}{ Computer gaming } & No gaming & 253 & $35.5^{v}$ & 747 & 55.7 & \multirow[t]{4}{*}{$\mathrm{X}^{2}(3, \mathrm{~N}=1873)=74.65, \mathrm{p}<0.001, \Phi \mathrm{c}=0.200$} & 156 & $41.0^{v}$ & 793 & 59.2 & \multirow[t]{4}{*}{$\mathrm{X}^{2}(3, \mathrm{~N}=1671)=41.95, \mathrm{p}<0.001, \Phi \mathrm{c}=0.158$} \\
\hline & Gaming without problems & 372 & $60.0^{\wedge}$ & 461 & 39.7 & & 180 & $52.0^{\wedge}$ & 443 & 36.9 & \\
\hline & Gaming with probable problems & 26 & 4.2 & 45 & 4.2 & & 27 & 7.0 & 44 & 3.7 & \\
\hline & Missing & 2 & $0.3^{\mathrm{a}}$ & 5 & $0.4^{\mathrm{a}}$ & & 0 & $0.0^{\mathrm{a}}$ & 1 & $0.2^{\mathrm{a}}$ & \\
\hline \multirow[t]{3}{*}{ Mental health ${ }^{\mathrm{d}}$} & Moderate to good mental health & 632 & 96.6 & 1203 & 95.8 & \multirow[t]{3}{*}{$\mathrm{X}^{2}(2, \mathrm{~N}=1875)=4.38, \mathrm{p}=0.112, \Phi \mathrm{c}=0.048$} & 346 & 95.3 & 1245 & 97.1 & \multirow[t]{3}{*}{$\mathrm{X}^{2}(2, \mathrm{~N}=1670)=8.65, \mathrm{p}=0.013, \Phi \mathrm{c}=0.072$} \\
\hline & Poor mental health & 18 & 3.0 & 34 & 2.7 & & 17 & 4.7 & 28 & 2.3 & \\
\hline & Missing & 3 & 0.4 & 21 & 1.5 & & 0 & $0.0^{\mathrm{a}}$ & 8 & 0.6 & \\
\hline
\end{tabular}

Observed cell count significantly higher $\left(^{\wedge}\right)$ or lower $\left(^{(}\right)$than expected $(p<0.01)$

${ }^{\text {a }}$ Expected cell count less than 5; $\Phi$ c estimate of effect size (Cramer's V).

b Land-based.

${ }^{\mathrm{c}}$ Measured with Alcohol Use Disorders Identification Test -Consumption. Cut-off score for risky consumption 5 and 6 for females and males, respectively.

d Measured with 5 item Mental Health Index. Cut-off score for poor perceived mental health is 52 .

\section{Table 3}

Gambling-related factors by gambling mode reported in column percentages (\%) for weighted sample and sample sizes (n) for unweighted sample.

\begin{tabular}{|c|c|c|c|c|c|c|c|c|c|c|c|}
\hline & & \multicolumn{5}{|c|}{ Males $(n=1911)$} & \multicolumn{5}{|c|}{ Females $(\mathrm{n}=1644)$} \\
\hline & & \multicolumn{2}{|c|}{$\begin{array}{l}\text { Online } \\
\text { gamblers } \\
(\mathrm{n}=653)\end{array}$} & \multicolumn{2}{|c|}{$\begin{array}{l}\mathrm{LB}^{\mathrm{b}} \\
\text { gamblers } \\
(\mathrm{n}=1258)\end{array}$} & \multirow[t]{2}{*}{$\mathrm{X}^{2}$ statistics } & \multicolumn{2}{|c|}{$\begin{array}{l}\text { Online } \\
\text { gamblers } \\
(\mathrm{n}=363)\end{array}$} & \multicolumn{2}{|c|}{$\begin{array}{l}\mathrm{LB}^{\mathrm{b}} \\
\text { gamblers } \\
(\mathrm{n}=1281)\end{array}$} & \multirow[t]{2}{*}{$\mathrm{X}^{2}$ statistics } \\
\hline & & $\mathrm{n}$ & $\%$ & $\mathrm{n}$ & $\%$ & & $\mathrm{n}$ & $\%$ & $\mathrm{n}$ & $\%$ & \\
\hline \multirow[t]{5}{*}{ Number of gambling types gambled } & 1 & 64 & $8.9^{\vee}$ & 428 & 33.0 & \multirow{5}{*}{$\mathrm{X}^{2}(4, \mathrm{~N}=1873)=468.42, \mathrm{p}<0.001, \Phi \mathrm{c}=0.500$} & 52 & $13.5^{\vee}$ & 503 & 39.1 & \multirow{5}{*}{$\mathrm{X}^{2}(4, \mathrm{~N}=1670)=308.62, \mathrm{p}<0.001, \Phi \mathrm{c}=0.430$} \\
\hline & 2 & 78 & $11.3^{\mathrm{v}}$ & 327 & 26.1 & & 77 & $20.8^{\mathrm{v}}$ & 457 & 35.2 & \\
\hline & 3 & 87 & $12.9^{2}$ & 243 & 19.3 & & 90 & $24.7^{\wedge}$ & 188 & 14.9 & \\
\hline & 4 & 86 & 13.1 & 135 & 11.0 & & 49 & $14.3^{\wedge}$ & 98 & 7.9 & \\
\hline & $5+$ & 338 & $53.8^{\wedge}$ & 125 & 10.6 & & 95 & $26.7^{\wedge}$ & 35 & 2.9 & \\
\hline \multirow[t]{3}{*}{ Expenditure by net income } & $<1 \%$ & 307 & 48.0 & 630 & 51.4 & \multirow{3}{*}{$\mathrm{X}^{2}(2, \mathrm{~N}=1873)=58.51, \mathrm{p}<0.001, \Phi \mathrm{c}=0.177$} & 203 & 57.4 & 718 & 56.6 & \multirow{3}{*}{$\mathrm{X}^{2}(2, \mathrm{~N}=1670)=68.07, \mathrm{p}<0.001, \Phi \mathrm{c}=0.202$} \\
\hline & $\geq 1 \%$ & 302 & $45.3^{\wedge}$ & 418 & 31.5 & & 129 & $34.0^{\wedge}$ & 262 & 18.7 & \\
\hline & Missing ${ }^{\mathrm{d}}$ & 44 & $6.7^{\vee}$ & 210 & 17.1 & & 31 & $8.6^{\vee}$ & 301 & 24.7 & \\
\hline \multirow[t]{4}{*}{$\mathrm{PGSI}^{\mathrm{c}}$} & Non-problem (0) & 471 & $71.0^{2}$ & 1095 & 86.9 & \multirow[t]{4}{*}{$\mathrm{X}^{2}(3, \mathrm{~N}=1873)=76.39, \mathrm{p}<0.001, \Phi \mathrm{c}=0.202$} & 296 & $81.0^{\circ}$ & 1187 & 92.5 & \multirow[t]{4}{*}{$\mathrm{X}^{2}(3, \mathrm{~N}=1670)=51.87, \mathrm{p}<0.001, \Phi \mathrm{c}=0.176$} \\
\hline & Low risk $(1-2)$ & 117 & $18.6^{\wedge}$ & 122 & 9.8 & & 50 & $13.8^{\wedge}$ & 80 & 6.3 & \\
\hline & Moderate risk (3-7) & 54 & $8.6^{\wedge}$ & 37 & 3.0 & & 12 & $3.6^{\wedge}$ & 13 & 1.1 & \\
\hline & Problem gambling $(\geq 8)$ & 11 & $1.8^{\wedge}$ & 4 & 0.3 & & 5 & $1.6^{\wedge a}$ & 1 & 0.1 & \\
\hline
\end{tabular}

Observed cell count significantly higher $\left(^{\wedge}\right)$ or lower $\left({ }^{(}\right)$than expected $(\mathrm{p}<0.01)$.

a Expected cell count less than 5; $\Phi c$ estimate of effect size (Cramer's V).

b Land-based.

Problem Gambling Severity Index.

${ }^{\mathrm{d}}$ Cases with no reported income or expenditure on gambling or reporting income of 0 . 
significant difference, adjusted residual method post hoc analyses were conducted to specify the differences. Explicitly, p-values were calculated from the adjusted residuals of individual cells obtained from the chi-square test, and these p-values were compared to Bonferroni corrected p-values at a significance level of 0.01 (MacDonald \& Gardner, 2000; Sharpe, 2015). Binary logistic regression models predicting online gambling were conducted for both genders separately with all previously utilized variables (Table 4). Number of gambling types gambled and PGSI and MHI-5 scores were entered into regression models as continuous variables. Sample weighting was used in all analyses to account for bias of non-responses, ensuring representativeness of the population by age, gender and living area (Salonen \& Raisamo, 2015). Statistical analyses were conducted with IBM SPSS Statistics version 22 (SPSS Inc., Chicago, Il, USA).

\section{Results}

\subsection{Group comparisons}

\subsubsection{Demographics and gambling mode}

The gender specific cross tabulation of demographic characteristics and mode of gambling (Table 1 ) yielded the following results: Online gamblers were generally younger than land-based gamblers. Among online gamblers there was a higher rate of 25-44 year olds $(\geq 21.5 \%)$ compared to rates seen among land-based gamblers $(\leq 14.8 \%)$ for both genders. Among the oldest age group (65-74 year olds) land-based gambling was more common regardless of gender. Among 55-64 year old males land-based gambling (22.5\%) was more common than online gambling (14.1\%).

There was a higher rate of singles among male online gamblers (49.5\%) compared to land-based gamblers (37.0\%), and likewise a higher rate of married individuals among male land-based gamblers (52.2\%) compared to their online gambling counterparts (42.2\%). Marital status was not associated to gambling mode among females.

Male online gamblers were significantly more likely to work full-time (63.6\%) compared to land-based gamblers (40.9\%). Male land-based gamblers were significantly more often age based pensioners $(23.2 \%)$ than online gamblers $(7.3 \%)$, with corresponding figures for females ( $22.2 \%$ vs. $13.0 \%$ ).

There was a significantly higher rate of individuals earning $1800-2499$ euros per month among male online gamblers $(29.8 \%)$ than male land-based gamblers (22.5\%), while there was a significantly higher rate of individuals earning 0-1199 euros per month among male land-based gamblers (18.9\%) compared to online gamblers (12.6\%). Net income was not associated to gambling mode among females.

\subsubsection{Risky behaviors, perceived mental health and gambling mode}

In the following set of chi-square analyses, risky behaviors and perceived mental health were compared between online and land-

Table 4

Binary logistic regression models predicting online gambling for males and females. Sample sizes ( $\mathrm{n}$ ) for unweighted sample.

\begin{tabular}{|c|c|c|c|c|c|c|c|c|c|}
\hline & & \multicolumn{4}{|c|}{ Male gamblers $(\mathrm{n}=1625)$} & \multicolumn{4}{|c|}{ Female gamblers $(\mathrm{n}=1299)$} \\
\hline & & B & Wald & sig. & OR $(\mathrm{CI} 95 \%)^{\mathrm{e}}$ & B & Wald & sig. & OR $(\mathrm{CI} 95 \%)^{\mathrm{e}}$ \\
\hline Constant & & -3.162 & 26.263 & $<0.001$ & 0.042 & -3.617 & 29.421 & $<0.001$ & 0.027 \\
\hline \multirow[t]{5}{*}{ Age group (ref. 18-24) } & $25-34$ & 0.556 & 4.442 & 0.035 & $\begin{array}{l}1.74(1.04 \\
-2.92)\end{array}$ & 0.740 & 5.624 & 0.018 & $\begin{array}{l}2.10(1.14 \\
-3.87)\end{array}$ \\
\hline & $35-44$ & 0.661 & 5.143 & 0.023 & $\begin{array}{l}1.94(1.09 \\
-3.43)\end{array}$ & 0.787 & 5.310 & 0.021 & $\begin{array}{l}2.20(1.13 \\
-4.29)\end{array}$ \\
\hline & $45-54$ & 0.773 & 6.363 & 0.012 & $\begin{array}{l}2.17(1.19 \\
-3.95)\end{array}$ & 0.366 & 1.037 & 0.309 & $1.44(0.71-2.92)$ \\
\hline & $55-64$ & 0.230 & 0.489 & 0.485 & $1.26(0.66-2.40)$ & -0.070 & 0.035 & 0.852 & $0.93(0.45-1.94)$ \\
\hline & $65-74$ & -0.162 & 0.122 & 0.727 & $0.85(0.34-2.12)$ & -0.350 & 0.438 & 0.508 & $0.71(0.25-1.99)$ \\
\hline \multirow[t]{7}{*}{ Employment status (ref. work full-time) } & Work part-time & -0.424 & 1.457 & 0.227 & $0.65(0.33-1.30)$ & -0.341 & 1.353 & 0.245 & $0.71(0.40-1.26)$ \\
\hline & Entrepeneur & -0.366 & 3.147 & 0.076 & $0.69(0.46-1.04)$ & 0.105 & 0.084 & 0.772 & $1.11(0.55-2.26)$ \\
\hline & Student & -0.295 & 0.957 & 0.328 & $0.75(0.41-1.34)$ & 0.374 & 1.327 & 0.249 & $1.45(0.77-2.75)$ \\
\hline & Unemployed/laid-off & -0.586 & 4.690 & 0.030 & $\begin{array}{l}0.56(0.33 \\
-0.95)\end{array}$ & 0.032 & 0.011 & 0.917 & $1.03(0.57-1.88)$ \\
\hline & Retired (age) & -0.533 & 2.641 & 0.104 & $0.59(0.31-1.12)$ & -0.005 & 0.000 & 0.989 & $1.00(0.47-2.10)$ \\
\hline & Retired (illness) & -0.467 & 1.751 & 0.186 & $0.63(0.31-1.25)$ & 0.239 & 0.382 & 0.536 & $1.27(0.60-2.71)$ \\
\hline & Homemaker & $\mathrm{N} / \mathrm{A}$ & & & & 0.285 & 0.766 & 0.381 & $1.33(0.70-2.52)$ \\
\hline \multirow[t]{2}{*}{ Marital status (ref. married) } & Single $\mathrm{a}^{\mathrm{a}}$ & 0.109 & 0.472 & 0.492 & $1.12(0.82-1.52)$ & -0.080 & 0.191 & 0.662 & $0.92(0.65-1.32)$ \\
\hline & Separated/widowed & -0.088 & 0.165 & 0.685 & $0.92(0.60-1.40)$ & 0.300 & 2.194 & 0.139 & $1.35(0.91-2.01)$ \\
\hline \multirow[t]{2}{*}{ Tobacco smoking (ref. no smoking) } & Occasional & -0.257 & 2.312 & 0.128 & $0.77(0.56-1.08)$ & 0.150 & 0.402 & 0.526 & $1.16(0.73-1.85)$ \\
\hline & Daily & -0.253 & 2.386 & 0.122 & $0.78(0.56-1.07)$ & -0.011 & 0.003 & 0.954 & $0.99(0.67-1.46)$ \\
\hline \multirow{2}{*}{$\begin{array}{l}\text { Alcohol consumption }{ }^{\mathrm{b}} \text { (ref. non-risky } \\
\text { consumption) }\end{array}$} & Non-consumption & 0.329 & 1.527 & 0.217 & $1.39(0.83-2.34)$ & 0.115 & 0.205 & 0.651 & $1.12(0.68-1.85)$ \\
\hline & Risky consumption & -0.112 & 0.596 & 0.440 & $0.89(0.67-1.19)$ & -0.151 & 0.478 & 0.489 & $0.86(0.56-1.32)$ \\
\hline \multirow[t]{2}{*}{ Computer gaming (ref. no gaming) } & Gaming without problems & 0.405 & 7.795 & 0.005 & $\begin{array}{l}1.50(1.13 \\
-1.99)\end{array}$ & 0.378 & 6.019 & 0.014 & $\begin{array}{l}1.46(1.08 \\
-1.97)\end{array}$ \\
\hline & Gaming with probable problems & 0.249 & 0.565 & 0.452 & $1.28(0.67-2.45)$ & 0.354 & 1.156 & 0.282 & $1.43(0.75-2.72)$ \\
\hline MHI- $5^{\mathrm{c}}$ sum score & & 0.005 & 0.895 & 0.344 & $1.01(0.99-1.02)$ & 0.005 & 0.641 & 0.423 & $1.01(0.99-1.02)$ \\
\hline Number of gambling types gambled & & 0.519 & 188.752 & $<0.001$ & $\begin{array}{l}1.68(1.56 \\
-1.81)\end{array}$ & 0.547 & 98.377 & $<0.001$ & $\begin{array}{l}1.73(1.55 \\
-1.93)\end{array}$ \\
\hline Expenditure by net income (ref. $<1 \%$ ) & $\geq 1 \%$ & 0.170 & 1.489 & 0.222 & $1.19(0.90-1.56)$ & 0.409 & 5.769 & 0.016 & $\begin{array}{l}1.51(1.08 \\
-2.10)\end{array}$ \\
\hline PGSI $^{\mathrm{d}}$ sum score & & 0.071 & 2.640 & 0.104 & $1.07(0.99-1.17)$ & 0.172 & 5.884 & 0.015 & $\begin{array}{l}1.19(1.03 \\
-1.37)\end{array}$ \\
\hline
\end{tabular}

Bold-faced rows indicate a statistically significant $(\mathrm{p}<0.05)$ predictor.

a Includes participants not in a registered relationship.

b Measured with Alcohol Use Disorders Identification Test -Consumption.

c 5 item Mental Health Inventory.

d Problem Gambling Severity Index.

e Odds ratio (95\% confidence interval). 
based gamblers for both genders separately (Table 2). There was partial evidence for an association between online gambling and tobacco smoking. Among males there was a higher rate $(\mathrm{p}<0.05)$ of non-smokers among land-based gamblers (60.5\%) compared to online gamblers (53.5\%). This trend was also evident among females ( $\mathrm{p}<0.05$ ), as $75.0 \%$ of land-based gamblers and $67.2 \%$ of online gamblers were non-smokers. Similarly a significant difference $(p<0.05)$ in rates of daily smokers was identified between female land-based gamblers (14.2\%) and online gamblers (20.1\%). Among males a higher proportion of online gamblers consumed alcohol at a risky level (36.1\%) compared to land-based gamblers (27.0\%). This same difference in rates was evident among females: $17.6 \%$ for online gamblers and $12.2 \%$ for land-based gamblers $(\mathrm{p}<0.05)$.

Compared to land-based gamblers, online gamblers were more likely to be gamers. $60.0 \%$ of male and $52.0 \%$ of female online gamblers engaged in gaming without problems, while $39.7 \%$ of male and $36.9 \%$ of female land-based gamblers engaged in gaming without problems. Non-gaming was significantly more common among land-based gamblers (55.7\% for males and $59.2 \%$ for females) compared to online gamblers (35.5\% and 41.0\%). Gaming with probable problems was not associated to the mode of gambling for either gender, nor was perceived mental health problems.

\subsubsection{Gambling-related factors and gambling mode}

Chi-square analyses for gambling related factors revealed that online gamblers tended to engage in a larger number of gambling types compared to land-based gamblers for both genders. A larger proportion of online gamblers (45.3\% for males and $34.0 \%$ for females) spent more than $1 \%$ of net income on gambling compared to land-based gamblers (31.5\% and 18.7\%). Online gamblers exhibited higher problem gambling scores than land-based gamblers regardless of gender. Non-problem gambling was significantly more common among land-based gamblers compared to online gamblers for both genders.

\subsection{Models predicting online gambling}

In the regression model for males, the following variables were significantly $(\mathrm{p}<0.05)$ associated to a higher probability of being an online gambler: age groups 25-34, 35-44 and 45-54 (compared to $18-24$ year olds); gaming without problems (compared to nongaming); and gambling on multiple gambling types. Additionally being unemployed or laid off significantly lowered the probability of being an online gambler compared to working full-time. Among females significant predictors of online gambling were: age groups 25-34 and 35-44; gaming without problems; gambling on multiple gambling types; expenditure amounting to over $1 \%$ of net income; and higher problem gambling scores. Results suggest that while controlling for the effects of the other variables inspected, marital status, tobacco smoking, risky alcohol consumption and perceived mental health problems were not significantly associated to mode of gambling for either gender.

Among males the logistic regression model was statistically significant $\left(X^{2}(24)=510.31, p<0.001\right)$, explaining over one third of variance of online gambling (Nagelkerke $\mathrm{R}^{2}=0.37$ ). The model classified $86.5 \%$ of male land-based gamblers and $60.2 \%$ of male online gamblers correctly. The model was also significant for females $\left(\mathrm{X}^{2}(24)=261.15\right.$, $\left.\mathrm{p}<0.001\right)$, explaining below one third of variance of online gambling (Nagelkerke $\mathrm{R}^{2}=0.26$ ). The model classified $94.3 \%$ of female land-based gamblers $34.9 \%$ of female online gamblers correctly. Hosmer and Lemeshow test indicates for both genders that the models fit data $(\mathrm{p}>0.05)$. There was no indication of multicollinearity (VIF values $<2$ and Tolerance $>0.5$ for all independent variables). With only one male gambler that was a homemaker, regression results could not be reported for this employment status.

\section{Discussion}

\subsection{Demographics}

In partial alignment with assumptions, regardless of gender online gamblers were proportionately more often younger than strictly land-based gamblers. Both participation in gambling and prevalence of problem gambling have increased among 18-24year-olds in Finland between 2011 and 2015 (Salonen \& Raisamo, 2015). However, present findings indicate that gambling online was most common among 25 to 44 year olds for both genders, while the model additionally suggested that males aged $45-54$ are more likely to gamble online compared to $18-24$ year olds. Differences in gambling tendencies among different age groups are likely to reflect general differences in prevailing life situations and in economic statuses, as discussed subsequently.

Firstly, 18-24 year olds are often students within the Finnish context, and this may influence their gambling behavior. In 2015 over $70 \%$ of the population aged $15 \leq$ years acquire education beyond primary and lower secondary level, such that among 20-29 year-olds $83 \%$ had attained at least upper secondary level education (OSF, 2016a). On completion of upper secondary education, individuals are about 18 years old, and hereafter $30-40 \%$ immediately continue studies at a higher level (Ministry of Education and Culture, 2016; OSF, 2016b). Internationally this age group typically presents high involvement in gambling practices (i.e. college students) (Barnes, Welte, Hoffman, \& Tidwell, 2010; Blinn-Pike, Worthy, \& Jonkman, 2007; St-Pierre, Temcheff, Gupta, Derevensky, \& Paskus, 2014). On the other hand as students tend to have limited income, their financial situation may cause them to refrain from gambling. In sum, online gambling among young adults needs to be investigated further.

Secondly, during the third to fifth decades of life individuals are often employed full-time, and thus are likely to have enough disposable income in order to gamble. Age related pension may not be granted in Finland before the age of 63 years, and the transitional age for age related pension in Finland is 63-68 years (Finnish Centre for Pensions, 2016; Rapo, 2014). As such, this age group is considered to partially be taking part in working life, but may also have more time and money for leisure activities, including gambling. It is also known that older adults favor casinos, slot machines and bingo as gambling activities (Tse, Hong, Wang, \& Cunningham-Williams, 2012), with distinct motivations to gamble (entertainment and leisure more than money and rewards) (Martin, Lichtenberg, \& Templin, 2011). Overall, online problem gambling studies conducted among older adults remain scarce (Sauvaget et al., 2015).

Age related differences in regard to online gambling probably also depict age group differences in the familiarity with necessary technologies to engage in online gambling. Between 2011 and 2015 Finnish online gambling increased in all age groups above 35-years (Salonen \& Raisamo, 2015), and gambling has been noted as a growing social activity among older adults (Subramaniam et al., 2015; Zaranek \& Chapleski, 2005). As practically the entire population aged 16-54 uses the internet, increases in the amount of users is evident only among older age groups (OSF, 2016c). For example, in $201692 \%$ of $55-64$ year olds and $74 \%$ of $65-74$ year olds used the internet (OSF, 2016c). Thus increasing rates of online gambling among older age groups may reflect the general rise in internet use among these age groups.

Being unemployed or laid-off was the only significant working 
status in the model, predicting land-based gambling among males. This stands in direct contrast to findings of Gainsbury et al. (2012). It may be that males without work are less likely to have disposable income on bank accounts or the possibility to gamble online due to faulty credit rating. Marital status was not associated to online gambling when controlling for other factors in the regression model. Taken together findings advocate that online gamblers are heterogeneous as suggested previously (Blaszczynski, Russell, Gainsbury, \& Hing, 2016), with some evident gender differences.

\subsection{Risky behaviors and perceived mental health}

Neither tobacco smoking or risky alcohol consumption were associated to online gambling in the regression models. The apparent higher degree of risky alcohol consumption and tobacco smoking among online gamblers indicated in chi-square analyses may be explicable by other confounding factors, potentially at-risk and problem gambling as recently suggested (Baggio et al., 2016). Consuming alcohol while gambling was most common among mixed mode gamblers (i.e. engaging in both online and land-based gambling), and least common among strictly online gamblers (Blaszczynski et al., 2016). Our results are in line with the aforementioned study, since online gamblers were largely representative of mixed mode gamblers. Interestingly, mixed-mode gamblers have been found to consume alcohol and tobacco less while gambling online than when gambling in land-based venues (Gainsbury, Russell, Hing, \& Wood 2014). Taken together, increased gambling may lead to increased alcohol consumption particularly among mixed mode gamblers.

Gaming without problems was clearly associated with online gambling which may imply that those who are accustomed to using computers tend to do so for a wide array of (leisure) activities. There may also be a more direct link between gambling and gaming, as the most common channels for encountering simulated gambling include popular video games (King et al., 2014), and bets have been found to be placed on computer games (Elton-Marshall et al., 2016). The fact that gaming with probable problems was not associated to gambling mode may be due to too few participants falling into this category, which in turn could be due to a low rate of self-recognition of problems. This null finding may also be due to measurement, since a single question was used instead of validated instrument. Poor perceived mental health was not associated to gambling mode at any point, suggesting that online gamblers do not perceive psychological problems more or less than land-based gamblers do. This is in line with the finding that mixed mode gamblers and land-based gamblers are at equivalent risk of experiencing psychosocial distress as proposed by Blaszczynski et al. (2016), who further suggested that strictly online gamblers were at lower risk. The gambling market in Finland is highly developed; in this context, as previously postulated by Wardle et al. (2011), online gambling may simply act as an additional mode of access for gambling. On the other hand our results are in conflict with the finding that online gamblers display higher rates of psychological distress (Gainsbury, Russell, Hing, Wood, et al., 2014), hence more research is needed on this.

\subsection{Gambling-related factors}

Online gamblers gambled on a larger number of gambling types than land-based gamblers. To elucidate results from the models, gamblers engaging in four gambling types were around four times as likely to be online gamblers as those gambling one gambling type. This same trend has been identified among adolescent gamblers (Elton-Marshall et al., 2016). The positive association between number of gambling types gambled and online gambling is likely due to both that beginning to gamble on an additional mode leads to an increase in number of gambling types, and that the resulting increased availability leads to increased gambling (Gainsbury et al., 2012). Importantly, previous research suggests the positive association between mixed-mode gambling and at-risk and problem gambling to be due to the elevated and more diverse participation among these gamblers (Baggio et al., 2016; Blaszczynski et al., 2016; Gainsbury, Russell, Blaszczynski, et al., 2015).

In the regression models, gambling expenditure amounting to over $1 \%$ of net income and gambling severity were associated with online gambling only among females, supporting findings that mixed-mode gamblers experience more gambling problems than strictly land-based or online gamblers (Blaszczynski et al., 2016; Elton-Marshall et al., 2016). Higher expenditure among female online gamblers may not be limited to those economically well-off, as income quartile was not associated to gambling mode. It has been suggested that mixed mode gamblers represent a subgroup of gamblers with some expendable income and few indispensable costs compared to single mode gamblers, whereby recreational gambling may lead to overspending and related problems (Gainsbury, Russell, Blaszczynski, et al., 2015).

There are several plausible explanations why online gambling may be particularly harmful among females. First, in contrast to males, females have been noted to prefer games of chance and gambling types that provide continuous forms of play (McCormack et al., 2014; Wardle et al., 2007). Compared to low event frequency games, high frequency online gambling types (e.g. online slot machines) may lead to higher expenditure (Auer \& Griffiths, 2016). Second, females view the internet as safe and less intimidating environment to gamble (McCormack et al., 2014). Third, females' choice to gamble online may be influenced by advertisements more strongly than among males (McCormack et al., 2014). Fourth, males possibly use responsible gambling tools more actively than females, which are readily available for online gambling environments (Blaszczynski et al., 2016). Fifth, from the view of the adaptation hypothesis (Abbott, 2006; Storer, Abbott, \& Stubbs, 2009), females may be in a different phase of adaptation (i.e. leveling may have already occurred for males). Females may be exposed to online gambling in an unpreceded manner, considering for example that marketing of online gambling towards females is a relatively novel phenomenon.

Finally, it's possible that social casino gaming is central to the female specific associations; as engagement in both activities is more common among females compared to males (Abarbanel \& Rahman, 2015) and migration from social casino gaming to online gambling has been linked to gambling problems (Gainsbury et al., 2016). Additionally, making in-game purchases in social casino games (or other freemium games) may lower the threshold to spend money on online gambling. In fact indulging in microtransactions was the sole predictor of migration from social casino gaming to online gambling in an online sample of social casino gamers (Kim, Wohl, Salmon, Gupta, \& Derevensky, 2014). The observed relationship between gaming and gambling may accordingly also be related to social casino games. Speculation concerning social casino games goes beyond what can be inferred from this study, but is a topical issue as online gambling and social casino gaming industries are converging (H2 Gambling Capital \& Odobo, 2013). It is important to realize that social casino games are often linked to Facebook, and companies seeking to migrate social casino gamers to online gambling may use profile information to pinpoint potential target groups (Abarbanel \& Rahman, 2015). Gaming and gambling need to be studied in more detail in the future, looking also into gaming genres, using more comprehensive measurement of problematic gaming (e.g. IGD-20) and longitudinal designs (Elton-Marshall et al., 2016; Pontes, Király, 
Demetrovics, \& Griffiths, 2014).

In January 2017, the three Finnish gambling service operators were merged into a single company. This merger may enhance the opportunities for responsible gambling, but detrimental consequences may also follow. This merger will lead to all online gamble services being provided from one single website, which may encourage individuals to engage in a wider variety of gambling types. Future research evaluating the impact of this merger is of chief importance, potentially providing insights on how to appropriately regulate gambling in cyberspace.

\subsection{Limitations}

This study was conducted utilizing data with clear merits, as gamblers were selected from a nationally representative survey sample with a high response rate and which was weighted for analyses. On the contrary, cross-sectional design hinders causal inferences to be made, and self-report is prone to bias. Importantly, self-reported amount of money spent gambling may be estimated incorrectly, particularly when actual expenditure is high (Auer \& Griffiths, 2016). Online gamblers also included gamblers who may have also participated in land-based gambling. This may have influenced results, as those who gamble both online and landbased are more likely to have gambling problems than the minority who exclusively gamble online (Gainsbury, Russell, Blaszczynski, et al., 2015). For the chi-square analyses appropriate post-hoc testing was conducted, but the effect sizes for the identified group differences were to a large extent small (Cohen, 1988), and may be considered trivial. Due to missing information on net income and gambling expenditure, several cases were omitted from the regression analyses. Gender differences seen in regression models are only conjectural, as odds ratio confidence intervals overlapped throughout (Gelman \& Stern, 2006). On the bright side, the subjective experience of gaming being problematic is an important aspect of gaming which has not been examined often, as is also the case for inspecting expenditure in relation to income. Importantly, few previous studies in this research field have examined gender specific correlates of online gambling.

\section{Conclusions}

Online gambling is a continuously expanding practice, and accordingly a greater understanding of online gambling needs to be gained. Previous findings are partially divergent on this subject, for example concerning how online gambling relates to demographic factors, gambling problems and substance use. This study builds upon this body of research by comparing the associations of online gambling and land-based gambling for males and females separately using a nationally representative sample.

Results show that in general online gamblers were younger than land-based gamblers among both genders. Nonetheless, online gambling is not limited to young adults, as it is encountered among individuals in their 50's, even more so than among 18 to 24 year olds. For both genders results indicate that online gamblers are typically experienced with computer gaming, and likewise that online gamblers engage in a higher number of gambling types than land-based gamblers. Risky alcohol consumption and tobacco smoking were not associated to gambling mode when controlling for other factors. Importantly, particularly for females online gambling may be related to higher relative expenditure and at-risk and problem gambling, providing implications for tailored interventions. In line with previous studies, it seems that the harmfulness of the internet as a mode of gambling is particularly related to the accompanied increased amount of gambling. The continued study of subgroups of gamblers is necessary to comprehensively understand the altering gambling milieu.

\section{Declaration of interest}

Authors have no actual or potential conflicts of interest that may inappropriately influence, or be perceived to influence, this work.

\section{Acknowledgments}

The authors would like to thank the Ministry of Social Affairs and Health (the $\S 52$ Appropriation of the Lotteries Act, contracts STM/3189/2011 \& STM/4743/2015) for funding this study. The funding source had no role in the conduct of the research or preparation of the article.

\section{References}

Abarbanel, B., \& Rahman, A. (2015). eCommerce market convergence in Action: Social casinos and real money gambling. UNLV Gaming Research \& Review Journal, 19(1), 51-62. http://digitalscholarship.unlv.edu/grrj/vol19/iss1/4/ (Accessed 17 November 2016).

Abbott, M. (2006). Do EGMs and problem gambling go together like a horse and carriage? Gambling Research, 18(1), 7-38. https://search.informit.com.au/ documentSummary; $\mathrm{dn}=836321688700553 ;$ res=IELFSC $\quad$ (Accessed 17 November 2016).

American Psychiatric Association. (2013). Diagnostic and statistical manual of mental disorders (5th ed.). Arlington, VA: Author (DSM-5).

Ariyabuddhiphongs, V. (2012). Older adults and gambling: A review. International Journal of Mental Health and Addiction, 10(2), 297-308. http://dx.doi.org/ 10.1007/s11469-011-9325-6.

Auer, M., \& Griffiths, M. D. (2016). Self-reported losses versus actual losses in online Gambling: An empirical study. Journal of Gambling Studies. http://dx.doi.org/ 10.1007/s10899-016-9648-0. Published online: 04 November 2016.

Baggio, S., Gainsbury, S. M., Berchtold, A., \& Iglesias, K. (2016). Co-morbidity of gambling and internet use among internet and land-based gamblers: Classic and network approaches. International Gambling Studies, 16(3), 500-517. http:// dx.doi.org/10.1080/14459795.2016.1242148.

Barnes, G. M., Welte, J. W., Hoffman, J. H., \& Tidwell, M.-C. O. (2010). Comparisons of gambling and alcohol use among college students and noncollege young people in the United States. Journal of American College Health, 58(5), 443-452. http:// dx.doi.org/10.1080/07448480903540499.

Blaszczynski, A., \& Nower, L. (2002). A pathways model of problem and pathological gambling. Addiction, 97, 487-499. http://dx.doi.org/10.1046/j.13600443.2002.00015.x.

Blaszczynski, A., Russell, A., Gainsbury, S., \& Hing, N. (2016). Mental health and online, land-based and mixed gamblers. Journal of Gambling Studies, 32(1), 261-275. http://dx.doi.org/10.1007/s10899-015-9528-z.

Blinn-Pike, L., Worthy, S. L., \& Jonkman, J. N. (2007). Disordered gambling among college students: A meta-analytic synthesis. Journal of Gambling Studies, 23, 175-183. http://dx.doi.org/10.1007/s10899-006-9036-2.

Bush, K., Kivlahan, D. R., McDonell, M. B., Fihn, S. D., \& Bradley, K. A. (1998). AUDIT alcohol consumption questions (AUDIT-C). Archives of Internal Medicine, 158(16), 1789-1795. http://dx.doi.org/10.1001/archinte.158.16.1789.

Castrén, S., Grainger, M., Lahti, T., Alho, H., \& Salonen, A. H. (2015). At-risk and problem gambling among adolescents: A convenience sample of first-year junior high school students in Finland. Substance Abuse Treatment, Prevention, and Policy, 10(1), 9. http://dx.doi.org/10.1186/s13011-015-0003-8.

Cohen, J. (1988). Statistical power analysis for the behavioral sciences (2nd ed.). Hillsdale, NJ: Erlbaum.

Corney, R., \& Davis, J. (2010). The attractions and risks of internet gambling for women: A qualitative study. Journal of Gambling Issues, 24, 121-139. http:// dx.doi.org/10.4309/2010.24.8.

Currie, S. R., Hodgins, D. C., Wang, J., El-Guebaly, N., Wynne, H., \& Chen, S. (2006) Risk of harm among gamblers in the general population as a function of level of participation in gambling activities. Addiction, 101(4), 570-580. http:// dx.doi.org/10.1111/j.1360-0443.2006.01392.x.

Dawson, D. A., Grant, B. F., Stinson, F. S., \& Zhou, Y. (2005). Effectiveness of the derived Alcohol Use Disorders Identification Test (AUDIT-C) in screening for alcohol use disorders and risk drinking in the US general population. Alcoholism, Clinical and Experimental Research, 29(5), 844-854. http://dx.doi.org/ 10.1097/01.ALC.0000164374.32229.A2.

Duven, E. C. P., Müller, K. W., Beutel, M. E., \& Wölfling, K. (2015). Altered reward processing in pathological computer gamers - ERP-results from a semi-natural Gaming-Design. Brain and Behavior, 5(1), 13-23. http://dx.doi.org/10.1002/ brb3.293.

Edgren, R., Castrén, S., Jokela, M., \& Salonen, A. H. (2016). At-risk and problem gambling among Finnish youth: The examination of risky alcohol consumption, tobacco smoking, mental health and loneliness as gender-specific correlates. Nordic Studies on Alcohol and Drugs, 33(1), 61-79. http://dx.doi.org/10.1515/ nsad-2016-0005. 
Elton-Marshall, T., Leatherdale, S. T., \& Turner, N. E. (2016). An examination of internet and land-based gambling among adolescents in three canadian provinces: Results from the youth gambling survey (YGS). BMC Public Health, 16(1), 277. http://dx.doi.org/10.1186/s12889-016-2933-0.

Ferguson, C. J., Coulson, M., \& Barnett, J. (2011). A meta-analysis of pathological gaming prevalence and comorbidity with mental health, academic and socia problems. Journal of Psychiatric Research, 45(12), 1573-1578. http://dx.doi.org/ 10.1016/j.jpsychires.2011.09.005.

Ferris, J., \& Wynne, H. (2001). The Canadian problem gambling Index: Final report Ottawa, ON: Canadian Centre on Substance Abuse. http://www.jogoremoto.pt/ docs/extra/TECb6h.pdf (Accessed 17 November 2016).

Finnish Centre for Pensions. (2016). Statistical yearbook of pensioners in Finland 2015. Helsinki: Juvenes Print - Suomen Yliopistopaino Oy. http://www.etk.fi/wpcontent/uploads/Tilasto_suomen_elakkeensaajista_2015.pdf (Accessed 28 January 2017).

Floros, G. D., Siomos, K., Fisoun, V., \& Geroukalis, D. (2013). Adolescent Online Gambling: The Impact of Parental Practices and Correlates with Online Activities. Journal of Gambling Studies, 29(1), 131-150. http://dx.doi.org/10.1007/ s10899-011-9291-8.

Gainsbury, S. M., Russell, A., Blaszczynski, A., \& Hing, N. (2015). The interaction between gambling activities and modes of access: A comparison of internetonly, land-based only, and mixed-mode gamblers. Addictive Behaviors, 41, 34-40. http://dx.doi.org/10.1016/j.addbeh.2014.09.023.

Gainsbury, S. M., Russell, A., \& Hing, N. (2014). An investigation of social casino gaming among land-based and internet gamblers: A comparison of sociodemographic characteristics, gambling and co-morbidities. Computers in $\mathrm{Hu}$ man Behavior, 33, 126-135. http://dx.doi.org/10.1016/j.chb.2014.01.031.

Gainsbury, S. M., Russell, A., Hing, N., Wood, R. T., \& Blaszczynski, A. (2013). The impact of internet gambling on gambling problems: A comparison of moderaterisk and problem internet and non-internet gamblers. Psychology of Addictive Behaviors, 27(4), 1092-1101. http://dx.doi.org/10.1037/a0031475.

Gainsbury, S. M., Russell, A., Hing, N., Wood, R., Lubman, D. I., \& Blaszczynski, A. (2014). The prevalence and determinants of problem gambling in Australia: Assessing the impact of interactive gambling and new technologies. Psychology of Addictive Behaviors, 28(3), 769-779. http://dx.doi.org/10.1037/a0036207.

Gainsbury, S. M., Russell, A., Hing, N., Wood, R. T., Lubman, D., \& Blaszczynski, A (2015). How the internet is changing gambling: Findings from an australian prevalence survey. Journal of Gambling Studies, 31(1), 1-15. http://dx.doi.org/ 10.1007/s10899-013-9404-7.

Gainsbury, S. M., Russell, A., King, D. L., Delfabbro, P., \& Hing, N. (2016). Migration from social casino games to gambling: Motivations and characteristics of gamers who gamble. Computers in Human Behavior, 63, 59-67. http:// dx.doi.org/10.1016/j.chb.2016.05.021.

Gainsbury, S. M., Wood, R. T., Russell, A., Hing, N., \& Blaszczynski, A. (2012). A digita revolution: Comparison of demographic profiles, attitudes and gambling behavior of Internet and non-Internet gamblers. Computers in Human Behavior, 28(4), 1388-1398. http://dx.doi.org/10.1016/j.chb.2012.02.024.

Gelman, A., \& Stern, H. (2006). The difference between "significant" and "not significant" is not itself statistically significant. The American Statistician, 60(4), 328-331. http://dx.doi.org/10.1198/000313006X152649.

Griffiths, M. D., \& Parke, J. (2010). Adolescent gambling on the internet: A review. International Journal of Adolescent Medicine and Health, 22(1), 59-75. http://usir. salford.ac.uk/id/eprint/18183.

H2 Gambling Capital \& Odobo. (2013). There's nothing virtual about the opportunity in real-money gambling: Opportunities for game developers in regulated realmoney online gambling. Gibraltar. http://docplayer.net/3323667-There-snothing-virtual-about-the-opportunity-gambling.html (Accessed 17 November 2016).

Hopley, A. A. B., \& Nicki, R. M. (2010). Predictive factors of excessive online poker playing. Cyberpsychology, Behavior and Social Networking, 13(4), 379-385. http://dx.doi.org/10.1089/cyber.2009.0223.

Kaarne, T., Aalto, M., Kuokkanen, M., \& Seppä, K. (2010). AUDIT-C, AUDIT-3 and AUDIT-QF in screening risky drinking among Finnish occupational health-care patients. Drug and Alcohol Review, 29(5), 563-567. http://dx.doi.org/10.1111 j.1465-3362.2010.00172.x

Kairouz, S., Paradis, C., \& Nadeau, L. (2012). Are online gamblers more at risk than offline gamblers? Cyberpsychology, Behavior and Social Networking, 15(3), 175-180. http://dx.doi.org/10.1089/cyber.2011.0260.

Kim, H. S., Wohl, M. J., Salmon, M. M., Gupta, R. \& Derevensky, J. (2014). Do social casino gamers migrate to online Gambling? An assessment of migration rate and potential predictors. Journal of Gambling Studies, 31(4), 1819-1831. http:/ dx.doi.org/10.1007/s10899-014-9511-0.

King, D. L., Delfabbro, P., Kaptsis, D., \& Zwaans, T. (2014). Adolescent simulated gambling via digital and social media: An emerging problem. Computers in Human Behavior, 31(1), 305-313. http://dx.doi.org/10.1016/j.chb.2013.10.048.

Király, O., Griffiths, M. D., Urban, R., Farkas, J., Kokonyei, G., Elekes, Z., et al. (2014) Problematic internet use and problematic online gaming are not the Same: Findings from a large nationally representative sample. Cyberpsychology Behaviour, and Social Networking, 17(12), 749-754. http://dx.doi.org/10.1089/ cyber.2014.0475.

Langham, E., Thorne, H., Browne, M., Donaldson, P., Rose, J., \& Rockloff, M. (2015). Understanding gambling related harm: A proposed definition, conceptual framework, and taxonomy of harms. BMC Public Health, 16(1), 80. http:// dx.doi.org/10.1186/s12889-016-2747-0.

Lotteries act [Arpajaislaki]. http://www.finlex.fi/fi/laki/ajantasa/2001/
20011047\#L2P5 (Accessed 02 August 2016).

MacDonald, P. L., \& Gardner, R. C. (2000). Type I error rate comparisons of post hoc procedures for $\mathrm{I} \times \mathrm{J}$ chi-square tables. Educational and Psychological Measurment, 60(5), 735-754. http://dx.doi.org/10.1177/00131640021970871.

Martin, F., Lichtenberg, P. A., \& Templin, T. N. (2011). A longitudinal Study: Casino gambling attitudes, motivations, and gambling patterns among urban elders. Journal of Gambling Studies, 27(2), 287-297. http://dx.doi.org/10.1007/s10899010-9202-4.

McBride, J., \& Derevensky, J. (2009). Internet gambling behavior in a sample of online gamblers. International Journal of Mental Health and Addiction, 7(1), 149-167. http://dx.doi.org/10.1007/s11469-008-9169-x.

McCormack, A., Shorter, G. W., \& Griffiths, M. D. (2014). An empirical study of gender differences in online gambling. Journal of Gambling Studies, 30(1), 71-88. http://dx.doi.org/10.1007/s10899-012-9341-x.

Ministry of Education and Culture. (2016). Education system in Finland. http://www. minedu.fi/export/sites/default/OPM/Koulutus/koulutusjaerjestelmae/liitteet/ finnish_education.pdf (Accessed 28 January 2017).

Official Statistics of Finland (OSF). (2016a). Educational structure of the population 2015 [e-publication]. Appendix table 1. Immediate transition to further studies of new passers of the matriculation examination 2005-2015. Helsinki: Statistics Finland. http://www.stat.fi/til/vkour/2015/vkour_2015_2016-11-03_en.pdf (Accessed 28 January 2017)

Official Statistics of Finland (OSF). (2016b). Entrance to education [e-publication]. Helsinki: Statistics Finland. http://www.stat.fi/til/khak/2015/khak_2015_201609-28_tau_001_en.html (Accessed 28 January 2017).

Official Statistics of Finland (OSF). (2016c). Finnish residents use the Internet more and more often [e-publication]. Helsinki: Statistics Finland. http://www.stat.fi/ til/sutivi/2016/sutivi_2016_2016-12-09_tie_001_en.html (Accessed 29 January 2017)

Orford, J., Wardle, H., Griffiths, M. D., Sproston, K., \& Erens, B. (2010). PGSI and DSMIV in the 2007 British gambling prevalence survey: Reliability, item response, factor structure and inter-scale agreement. International Gambling Studies, 10(1), 31-44. http://dx.doi.org/10.1080/14459790903567132.

Petry, N. M., \& O'Brien, C. P. (2013). Internet gaming disorder and the DSM-5. Addiction, 108(7), 1186-1187. http://dx.doi.org/10.1111/add.12162.

Petry, N. M., \& Weinstock, J. (2007). Internet gambling is common in college students and associated with poor mental health. The American Journal on Addictions, 16(5), 325-330. http://dx.doi.org/10.1080/10550490701525673.

Pontes, H. M., Király, O., Demetrovics, Z., \& Griffiths, M. D. (2014). The conceptualisation and measurement of DSM-5 internet gaming disorder: The development of the IGD-20 test. PLoS ONE, 9(10). http://dx.doi.org/10.1371/ journal.pone.0110137.

Rapo, M. (2014). Ikärakenne pakottaa keskustelemaan eläkeiästä [The changing age structure: Implications for retirement age]. Article published by Statistics Finland, Hyvinvointikatsaus 2/2014 http://www.stat.fi/artikkelit/2014/art_2014-05-26_ 001.html (Accessed 28 January 2017).

Rumpf, H. J., Meyer, C., Hapke, U., \& John, U. (2001). Screening for mental health: Validity of the MHI-5 using DSM-IV Axis I psychiatric disorders as gold standard. Psychiatry Research, 105(3), 243-253. http://dx.doi.org/10.1016/S01651781(01)00329-8.

Salonen, A. H., Alho, H., \& Castrén, S. (2016). The extent and type of gambling harms for concerned significant others: A cross-sectional population study in Finland. Scandinavian Journal of Public Health, 44, 799-804. http://dx.doi.org/10.1177/ 1403494816673529.

Salonen, A. H., \& Raisamo, S. (2015). Suomalaisten rahapelaaminen 2015Rahapelaaminen, rahapeliongelmat ja rahapelaamiseen liittyvät asenteet ja mielipiteet 15-74-vuotiailla [Finnish Gambling 2015-Gambling, gambling problems and gambling related attitudes and opinions among 15-74 year-olds]. http://www. julkari.fi/handle/10024/129595 (Accessed 17 November 2016).

Sauvaget, A., Jiménez-Murcia, S., Fernández-Aranda, F., Fagundo, A. B., Moragas, L., Wolz, I., et al.Menchón, J. M. (2015). Unexpected online gambling disorder in late-life: A case report. Frontiers in Psychology, 6(655), 1-9. http://dx.doi.org/ 10.3389/fpsyg.2015.00655

Scholes-Balog, K. E., \& Hemphill, S. A. (2012). Relationships between online gambling, mental health, and substance use: A review. Cyberpsychology, Behavior, and Social Networking, 15(12). http://dx.doi.org/10.1089/ cyber.2012.0232, 121019105613009.

Sharpe, D. (2015). Your chi-square test is statistically significant: Now what? Practical Assessment, Research and Evaluation, 20(8), 1-10. http://www. pareonline.net/getvn.asp? $\mathrm{v}=20 \& \mathrm{n}=8$ (Accessed 19 November 2016).

St-Pierre, R. A., Temcheff, C. E., Gupta, R., Derevensky, J., \& Paskus, T. S. (2014). Predicting gambling problems from gambling outcome expectancies in college student-athletes. Journal of Gambling Studies, 30, 47-60. http://dx.doi.org/ $10.1007 / \mathrm{s} 10899-012-9355-4$

Storer, J., Abbott, M., \& Stubbs, J. (2009). Access or adaptation? A meta-analysis of surveys of problem gambling prevalence in Australia and New Zealand with respect to concentration of electronic gaming machines. International Gambling Studies, 9(3), 225-244. http://dx.doi.org/10.1080/14459790903257981.

Strand, B. H., Dalgard, O. S., Tambs, K., \& Rognerud, M. (2003). Measuring the mental health status of the norwegian population: A comparison of the instruments SCL-25, SCL-10, SCL-5 and MHI-5 (SF-36). Nordic Journal of Psychiatry, 57(2), 113-118. http://dx.doi.org/10.1080/08039480310000932.

Subramaniam, M., Wang, P., Soh, P., Vaingankar, J., Chong, S., Browning, C., et al. (2015). Prevalence and determinants of gambling disorder among older adults: A systematic review. Addictive Behaviors, 41, 119-209. http://dx.doi.org/10.1016/ 
j.addbeh.2014.10.007.

Svensson, J., \& Romild, U. (2011). Incidence of internet gambling in Sweden: Results from the swedish longitudinal gambling study. International Gambling Studies, 11(3), 37-41. http://dx.doi.org/10.1080/14459795.2011.629203.

Tse, S., Hong, S., Wang, C., \& Cunningham-Williams, R. M. (2012). Gambling behavior and problems among older adults: A systematic review of empirical studies. Journals of Gerontology Series B: Psychological Sciences and Social Sciences, 67(5), 639-652. http://dx.doi.org/10.1093/geronb/gbs068.

Veikkaus. (2014). Social responsibility report and annual report 2013. http://www. veikkaus2013.fi/fi/tilinpaatos/hallituksen-toimintakertomus-vuodelta-2013/ (Accessed 03 August 2016).

Veikkaus. (2016). Annual report 2015. https://cms.veikkaus.fi/site/binaries/content/ assets/dokumentit/vuosikertomus/2015/vuosiraportti_2015_suomi.pdf (Accessed 03 August 2016).

Veit, C. T., \& Ware, J. E. (1983). The structure of psychological distress and wellbeing in general populations. Journal of Consulting and Clinical Psychology, 51(5), 730-742. http://dx.doi.org/10.1037/0022-006X.51.5.730.

Wardle, H., Moody, A., Griffiths, M. D., Orford, J., \& Volberg, R. (2011). Defining the online gambler and patterns of behaviour integration: Evidence from the British gambling prevalence survey 2010. International Gambling Studies, 11(3), 339-356. http://dx.doi.org/10.1080/14459795.2011.628684.

Wardle, H., Sproston, K., Orford, J., Erens, B., Griffiths, M., Constantine, R., et al (2007). British gambling prevalence survey 2007. National Centre for Social Research.

Weinstock, J., Whelan, J. P., \& Meyers, A. (2008). College Students ' Gambling Behavior: When Does It Become Harmful? Journal of American College Health, 56(5), 513-521. http://dx.doi.org/10.3200/JACH.56.5.513-522.

Wood, R. T., Griffiths, M. D., \& Parke, J. (2007). Acquisition, development, and maintenance of online poker playing in a student sample. Cyberpsychology \& Behavior, 10(3), 354-361. http://dx.doi.org/10.1089/cpb.2006.9944.

Wood, R. T., \& Williams, R. J. (2011). A comparative profile of the Internet gambler: Demographic characteristics, game-play patterns, and problem gambling status. New Media \& Society, 13(7), 1123-1141. http://dx.doi.org/10.1177| 1461444810397650.

Zaranek, R. R., \& Chapleski, E. E. (2005). Casino gambling among urban elders: Just another social activity? Journal of Gerontology, 60B(2), S74-S81. 